\title{
Separation of Interfering Signals in an Ultrasonic Flow Measurement System by Using Variable Time-Delay Properties
}

\author{
Bächle, Matthias \\ Institute of Industrial Inform. Techn. \\ Karlsruhe Institute of Technology \\ Karlsruhe, Germany \\ https://orcid.org/0000-0001-7228-299X
}

\author{
Heizmann, Michael \\ Institute of Industrial Inform. Techn. \\ Karlsruhe Institute of Technology \\ Karlsruhe, Germany \\ michael.heizmann@kit.edu
}

\begin{abstract}
The accuracy of ultrasonic flow measurements based on the transit-time principle is highly influenced by the level of superimposed interfering signals caused, among other things, by a dispersive multipath propagation across the pipe wall. This leads to an interference in the same frequency and time domain, preventing the use of conventional filtering. To avoid expensive constructive methods for suppressing the interfering signals, we present a digital signal processing approach that uses the different characteristics of the desired and the interfering signals. In this work, the variable time-delay property of the fluid signal is used. Thus, the temperature-dependent speed of sound and a varying flow velocity are exploited. To this end, multiple measurements with varying temperature and flow velocity are recorded and then processed to estimate the static interfering signals. The results are evaluated using the estimation error of the transit-time difference in a simulated and an experimental flow measurement scenario, respectively.
\end{abstract}

Index Terms - ultrasonic flow measurement, interfering signals, B-Splines

\section{INTRODUCTION}

Ultrasonic flow measurements using the transit-time principle are widespread in industrial applications due to their advantages such as low energy consumption, low maintenance cost and non-intrusiveness of the measurement [1], [2]. However, additive interfering signals, induced by crosstalk through the pipe wall, can lead to a reduced accuracy, if they arise at the same time as the desired signals [3]. Since the interfering signals are caused by a multipath propagation, they are highly correlated with the desired signals, and therefore hard to separate by using signal characteristics such as frequency range. While Lynnworth and Liu [1] report only mechanical methods for crosstalk damping, Roosnek [4] and Jacobson et al. [5] use the dynamics of the fluid signals to separate them by post-processing. Nevertheless, Roosnek [4] needs the flow statistics to create time-delays of the fluid signals which uniformly cover one periodic time in order to use the destructive interference for estimation of the non-varying crosstalk. On the other hand, Jacobson et al. [5] realize the suppression of the non-varying interfering signals by using a high-pass along a set of consecutive measurements. This in turn needs the dynamic properties of the fluid signals to be sufficiently distinct. Thus, in our previous publication [6] we proposed a method to use even small variations of the time delays to get an interference-invariant measurement of the transit-time. However, the assumption of a constant flow velocity during the consecutive measurements was necessary. In this work, this constraint will be alleviated to allow a robust estimation, even if the flow velocity is varying during the consecutive measurements.

\section{Methodology}

The separation of the additive interfering signals is realized by estimating and then simply subtracting them. For this purpose, we exploit the property that the interfering signals are static and independent of the temperature $T$ and the flow velocity $v_{\mathrm{F}}$. To model these effects, the basic model

$$
\begin{aligned}
& y_{\mathrm{u}}(t ; T, v)=x\left(t-t_{\mathrm{d}}\left(v_{\mathrm{F}}\right) / 2-t_{\mathrm{a}}(T)\right)+e(t)+n_{1}(t) \\
& y_{\mathrm{d}}(t ; T, v)=x(t+\underbrace{t_{\mathrm{d}}\left(v_{\mathrm{F}}\right) / 2-t_{\mathrm{a}}(T)}_{\tau})+e(t)+n_{2}(t)
\end{aligned}
$$

is composed of the direct signals through the fluid $x(t)$, the interfering signals $e(t)$ and the Additive White Gaussian Noise (AWGN) $n(t)$. These together build the upstream and downstream measurement signals $y_{\mathrm{u}}(t ; T, v)$ and $y_{\mathrm{d}}(t ; T, v)$. Here, the flow velocity $v_{\mathrm{F}}$ influences the transit-time difference $t_{\mathrm{d}}\left(v_{\mathrm{F}}\right)$ and the absolute time-delay $t_{\mathrm{a}}(T)$ is determined by the temperature-dependent speed of sound $c_{\mathrm{F}}(T)$, if water is the medium. These variable time-delay properties of the direct signals can be used to formulate the following derivations. For our algorithm, either the upstream or downstream signals are necessary, which is why the following calculations only consider the downstream signals, where the subscripts are omitted.

Suppose there are $M$ consecutive measurements with varying flow velocity $v_{\mathrm{F}, m}$ and temperature $T_{m}$. These process parameter variations result in a varying time delay $\tau$, which covers the interval $\tau \in[\underline{t}, \bar{t}]$. Thus, at any two time steps $t_{n}$ and $t_{n+1}$, there exist sample points of the direct signals $x(t)$ in the intervals $\Gamma_{n}=\left[t_{n}+\underline{t}, t_{n}+\bar{t}\right]$ and $\Gamma_{n+1}=\left[t_{n+1}+\underline{t}, t_{n+1}+\bar{t}\right]$, respectively. If the sampling time is sufficiently small, the 
intersection $\Gamma=\Gamma_{n} \cap \Gamma_{n+1}$ is non-empty and for every $\left(\tau_{1}+t_{n}\right) \in \Gamma$, there is exactly one $\left(\tau_{2}+t_{n+1}\right) \in \Gamma$ such that

$$
\tilde{y}\left(t_{n} ; \tau_{1}\right)+\Delta \tilde{e}\left(t_{n}\right)+\Delta \tilde{n}\left(t_{n}\right)=\tilde{y}\left(t_{n+1} ; \tau_{2}\right),
$$

with

$$
\begin{aligned}
\Delta \tilde{e}\left(t_{n}\right) & =\tilde{e}\left(t_{n+1}\right)-\tilde{e}\left(t_{n}\right), \\
\Delta \tilde{n}\left(t_{n}\right) & =\tilde{n}\left(t_{n+1}\right)-\tilde{n}\left(t_{n}\right)
\end{aligned}
$$

holds. In this paper, the notation $(\tilde{\cdot})$ denotes the analytic signal calculated by the sum of the signal itself and its complexvalued Hilbert transform $\mathrm{j} \mathcal{H}\{\cdot\}$. Note that in Eq. (2) the parameters $v_{\mathrm{F}}$ and $T$ have been replaced by their resulting time-delays $\tau_{1}$ and $\tau_{2}$.

Equation(2) shows that the interfering signals can be estimated by finding all corresponding time-delays $\tau_{1}, \tau_{2}$ to resolve the formula to $\Delta \tilde{e}\left(t_{n}\right)$. However, since the time-delays contained in the data set of the $M$ measurement signals are only influenced by the process parameters $v_{\mathrm{F}, m}, T_{m}$, the distribution in the interval $\Gamma$ may be non-uniform or even contain gaps. Therefore, some time-delays $\tau_{1}$ do not have a corresponding time-delay $\tau_{2}$ in the data set. To circumvent this issue and to be more robust against AWGN (see Eq. (4)), we propose to use approximations of the analytic signals $\tilde{y}\left(t_{n} ; \tau_{1}\right)$ and $\tilde{y}\left(t_{n+1} ; \tau_{2}\right)$ based on B-Splines. This results in two trajectories $\hat{\mathbf{p}}_{n}(r)$ and $\hat{\mathbf{p}}_{n+1}(r)$. After the B-Spline based approximation of the signals, the differential interfering signals $\Delta \tilde{e}\left(t_{n}\right)$ is estimated by calculating the misalignment between the overlapping parts of the two trajectories $\hat{\mathbf{p}}_{n}(r), \hat{\mathbf{p}}_{n+1}(r)$. This part of the algorithm is called Partial Shape Matching.

In the first step of the approximation via B-Splines, a point cloud

$$
\mathbf{P}_{n}=\left[\mathbf{p}_{n, 1}, \ldots, \mathbf{p}_{n, M}\right]^{\mathrm{T}} \in \mathbb{R}^{M \times 2},
$$

consisting of the $2 \mathrm{D}$ representation of the analytic measurements signals at the time step $t_{n}$

$$
\mathbf{p}_{n, m}=\left[\begin{array}{l}
\operatorname{Re}\left\{\tilde{y}\left(t_{n} ; T_{m}, v_{m}\right)\right\} \\
\operatorname{Im}\left\{\tilde{y}\left(t_{n} ; T_{m}, v_{m}\right)\right\}
\end{array}\right] \in \mathbb{R}^{2 \times 1},
$$

is created, which results in $M$ 2D points (for a detailed description of the point cloud generation see [6]). A 2D trajectory can be modeled by

$$
\hat{\mathbf{p}}(r)=\sum_{i=1}^{F} b_{i}(r) \cdot \mathbf{c}_{i} \quad, \quad \mathbf{c}_{i}=\left[\begin{array}{c}
c_{x, i} \\
c_{y, i}
\end{array}\right],
$$

where $b_{i}(r)$ denotes the B-Splines of order $L$ and $\mathbf{c}_{i}$ denotes the coefficients of the $F$ individual B-Splines. $F$ represents the degrees of freedom, which can be increased by using more support knots or higher-order B-Splines, if the approximation quality is insufficient. The approximation problem can then be formulated as

$$
\mathbf{P}_{n}=\mathbf{B}(\mathbf{r}) \cdot \mathbf{C}+\mathbf{n}_{n} \quad, \quad \mathbf{C}=\left[\mathbf{c}_{1}, \ldots, \mathbf{c}_{F}\right]^{\mathrm{T}} \in \mathbb{R}^{F \times 2},
$$

with the corresponding support points

$$
\mathbf{r}=\left[r_{1}, \ldots, r_{M}\right]^{\mathrm{T}} \quad, \quad r_{m} \in[0,1]
$$
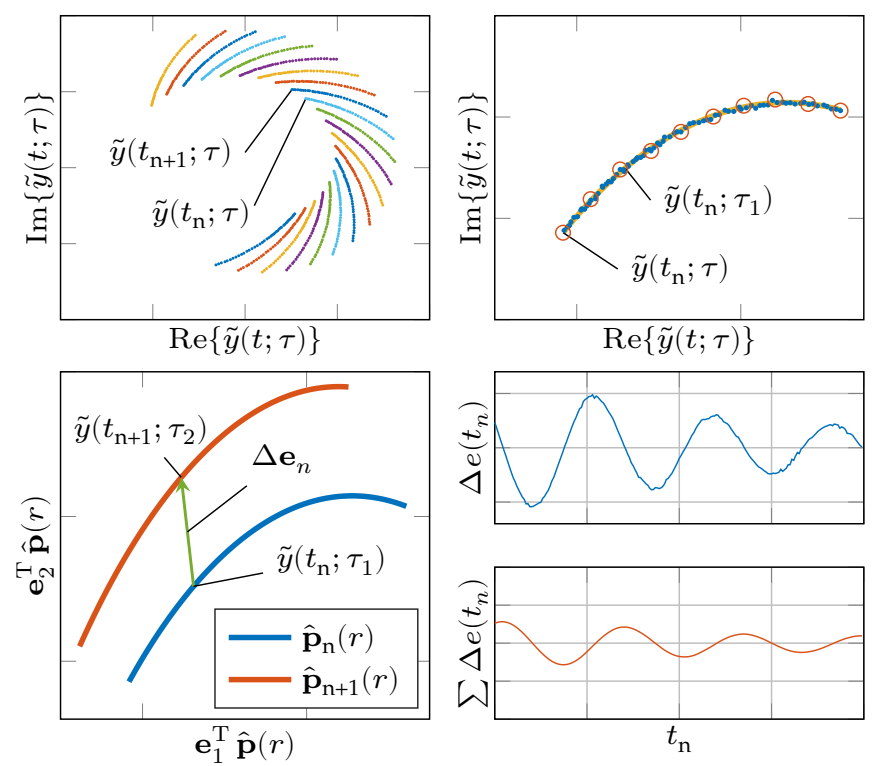

Fig. 1. Example estimation of the interfering signals. Upper left: all point clouds. Upper right: B-Spline approximation of a single point cloud. Lower left: partial shape matching between two trajectories. Lower right: estimated differential interfering signals $\Delta e\left(t_{\mathrm{n}}\right)$ and their offset corrected integration.

and the resulting B-Spline matrix

$$
\mathbf{B}(\mathbf{r})=\left[b_{1}(\mathbf{r}), \ldots, b_{F}(\mathbf{r})\right] \in \mathbb{R}^{M \times F} .
$$

The scaling of the support points $r$ can be arbitrarily chosen, because the points to approximate are only given as $2 \mathrm{D}$ vectors, but the scaling needs to be adapted to the interval spanned by the support knots. For simplicity, we set the scaling of the support points and correspondingly the support knots to the interval $[0,1]$. After the formulation of the problem, the approximation can be performed by the iterative method of Wang et al. [7]:

$$
\left\{\begin{array}{l}
\mathbf{r}^{(0)}: \quad \text { initial support points } \\
\mathbf{C}^{(k+1)}=\left(\mathbf{B}^{\mathrm{T}}\left(\mathbf{r}^{(k)}\right) \mathbf{B}\left(\mathbf{r}^{(k)}\right)\right)^{-1} \mathbf{B}^{\mathrm{T}}\left(\mathbf{r}^{(k)}\right) \cdot \mathbf{P}_{n} \\
\mathbf{r}^{(k+1)}=\arg \min _{\mathbf{r}}\left\|\mathbf{B}(\mathbf{r}) \cdot \mathbf{C}^{(k)}-\mathbf{P}_{n}\right\|_{F}^{2} .
\end{array}\right.
$$

Finding the initial support points is realized by pre-ordering the points $\mathbf{p}_{n, m}$ using the Euclidean distance. Since the trajectories do not have loops and are only slightly curved (see Fig. 1), the two points with maximal distance between each other represent the start and end points. The remaining support points are determined by their distance to the start point with subsequent normalization of all distances to the interval $[0,1]$.

Subsequently to the B-Spline approximation, the misalignment has to be estimated. For this purpose, both trajectories $\hat{\mathbf{p}}_{n}(r), \hat{\mathbf{p}}_{n+1}(r)$ are densely sampled and a quality measure using the mean of squared distances is introduced. Because only a partial overlap is expected, the sampled points of the first trajectory are classified whether they have a corresponding point in the other trajectory or not. All points with 
corresponding counterparts are connected using the nearest neighbor search and their squared distances are accumulated in the quality measure. By simple minimization of the quality measure, the misalignment $\Delta \tilde{e}\left(t_{n}\right)$ can then be determined.

However, an individual B-Spline approximation for every time step $t_{n}$ leads to slight deviations in the shapes of the trajectories due to the noise $\mathbf{n}$, which induces further errors when estimating the misalignment. A better approach is the combined B-Spline and misalignment approximation, as it integrates the knowledge that the shapes of the trajectories at different time steps do not change. Furthermore, the computational effort used for Partial Shape Matching is saved. The combined estimation can be achieved by extending the signal model (8) by the misalignment, which results in the adapted formulation

$$
\underbrace{\left[\begin{array}{c}
\mathbf{P}_{n} \\
\mathbf{P}_{n+1}
\end{array}\right]}_{\hat{\mathbf{P}}_{n}}=\underbrace{\left[\begin{array}{cc}
\mathbf{B}\left(\mathbf{r}_{n}\right) & \mathbf{0} \\
\mathbf{B}\left(\mathbf{r}_{n+1}\right) & \mathbf{I}
\end{array}\right]}_{\hat{\mathbf{B}}(\hat{\mathbf{r}})} \cdot\left[\begin{array}{c}
\mathbf{C} \\
\Delta \mathbf{e}_{n}^{\mathrm{T}}
\end{array}\right]+\left[\begin{array}{c}
\mathbf{n}_{n} \\
\mathbf{n}_{n+1}
\end{array}\right],
$$

with the ones matrix

$$
\mathbf{I}=[1, \ldots, 1]^{\mathrm{T}} \in \mathbb{R}^{M \times 1}
$$

and the misalignment

$$
\Delta \mathbf{e}_{n}=\left[\begin{array}{l}
\operatorname{Re}\left\{\tilde{e}\left(t_{n+1}\right)-\tilde{e}\left(t_{n}\right)\right\} \\
\operatorname{Im}\left\{\tilde{e}\left(t_{n+1}\right)-\tilde{e}\left(t_{n}\right)\right\}
\end{array}\right] .
$$

This way, the iterative approach (11) can be reused with the modified point cloud $\hat{\mathbf{P}}_{n}$ and the modified B-Spline matrix $\hat{\mathbf{B}}(\hat{\mathbf{r}})$.

Finally, after the repeated estimation of $\Delta \tilde{e}\left(t_{n}\right)$ for all relevant time steps $t_{n}$, the differential interfering signal needs to be integrated to separate it from the measurement signals. The integration constant is determined by an offset correction, which is justified by the band pass characteristics of the interfering signals. An example overview over the different algorithm steps, such as point cloud generation, B-Spline approximation, misalignment estimation and integration can be seen in Fig. 1.

\section{Simulation AND ExPERIMENTAL Results}

For the detailed evaluation of the new method and its dependencies, simulated signals were generated. The underlying simulation environment used the pipe geometry of our experiment (diameter $8 \mathrm{~cm}$ and axial transducer distance $6 \mathrm{~cm}$ ) and considered effects such as temperature dependency of the speed of sound in water and the flow velocity averaged over the propagation path. The signals were generated according to (1) and consisted of direct signals, additive interfering signals and measurement noise. As direct signals a cosine modulated Gaussian impulse with a center frequency of $700 \mathrm{kHz}$ was used. And as additive interfering signals, we recorded a typical signal from an air-filled pipe using clamp-on transducers to get realistic characteristics. Lastly, the measurement noise was simulated by the MATLAB random numbers generator and adjusted to test the effect of different Signal-to-NoiseRatios (SNR). Due to the interfering signals being known,
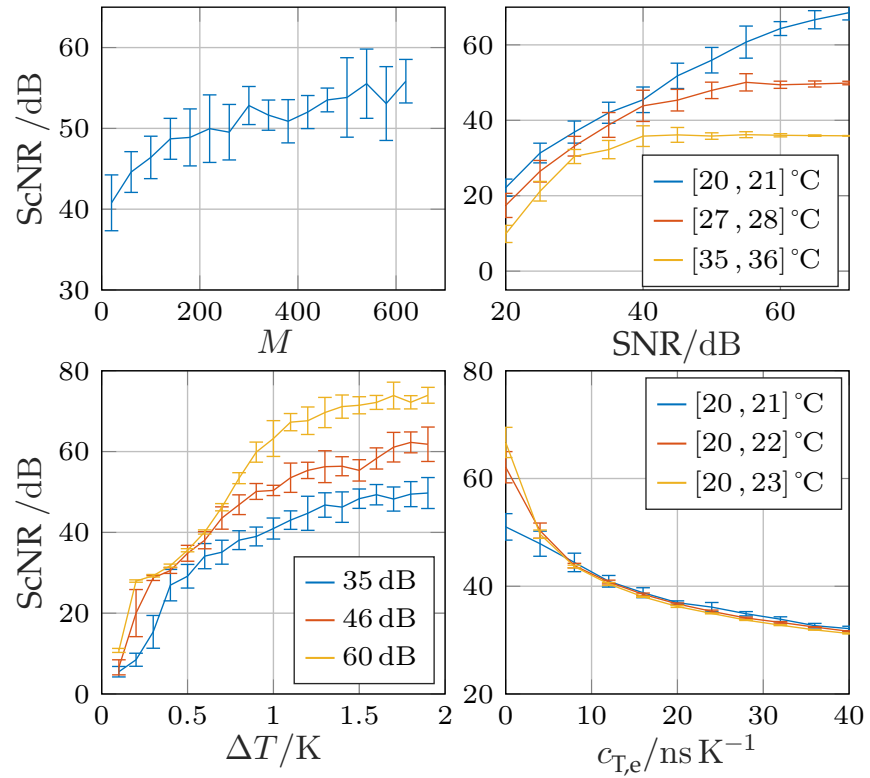

Fig. 2. Evaluation of estimation quality using different simulated scenarios. Upper left: Dependency on the number of measurements $M(\mathrm{SNR}=46 \mathrm{~dB}$, $\Delta T=1 \mathrm{~K})$. Upper right: Dependency on the noise level $(M=400)$. Lower left: Dependency on the temperature dynamic range $[20,20+\Delta T]^{\circ} \mathrm{C}$. Lower right: Limitation of the quality by non-static interfering signals.

the estimation quality can then be evaluated by the residual Signal-to-correlated-Noise-Ratio

$$
\operatorname{ScNR}=20 \cdot \log \left(\frac{\max \left|x\left(t_{n}\right)\right|}{\left\|\hat{e}\left(t_{n}\right)-e\left(t_{n}\right)\right\|_{2}}\right),
$$

where $\hat{e}\left(t_{n}\right)$ denotes the estimated interfering signal. Note that the maximum of the direct signal was normalized to 1 , which simplifies the above equation.

Since the proposed method is based on the signal model (1) and requires $M$ consecutive measurements with variable timedelay properties, different scenarios with varying numbers of measurements $M$, temperature dynamic ranges $\Delta T$ over the $M$ measurements, SNR conditions and model errors were simulated. Due to the assumption of static interfering signals, we introduced a temperature-dependent time-delay $t_{\mathrm{e}}(T)$ to model different levels of model errors. For reasons of simplicity, the linear relation $t_{\mathrm{e}}(T)=c_{\mathrm{T}, \mathrm{e}}\left(T-20^{\circ} \mathrm{C}\right)$ was used to model the temperature dependency. In Fig. 2 the estimation quality using the metric (15) is depicted for different scenarios. It can be seen, that the estimation quality is impaired by higher temperatures, worse SNR levels and the model error. However, the number of measurements does not significantly influence the quality, since the ScNR values are still above $40 \mathrm{~dB}$, which is required to get an estimation of the transittime difference with an accuracy of $\pm 1 \%$ [6]. Furthermore, a minimum temperature dynamic range of $\Delta T=0.7 \mathrm{~K}$ is necessary for the method to be robust against AWGN. Lastly, Fig. 2 shows that the model error of non-static interfering signals dominates the estimation quality, because even larger temperature dynamic ranges can not improve the estimation. 


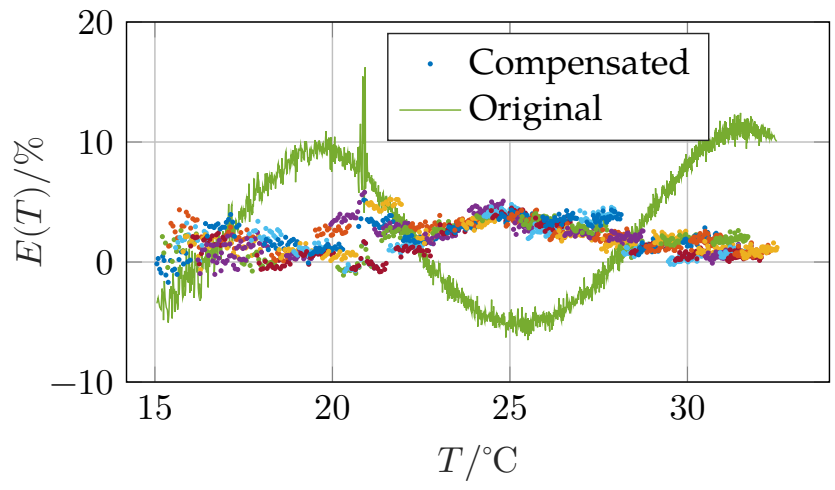

Fig. 3. Comparison of the transit-time difference errors if the original or interference-compensated signals were used.

The experimental results were obtained from an ultrasonic flow measurement experiment, which consisted of a water cycle with a pump, a reference measurement device and a heating element installed. Over a constantly increasing temperature, 2261 measurements with varying flow velocity were recorded and reordered in several signal packages, which cover a temperature dynamic range of $\Delta T=1 \mathrm{~K}$ each. The packages were chosen to contain overlapping temperature ranges, in order to visualize discontinuities if they exist. Because the ground-truth for the interfering signals is not known, the quality of the compensation is evaluated by the resulting estimation quality of the transit-time difference. Therefore, the transit-time estimation method introduced by Kupnik et al. [8] is applied once to the original signals and once to the compensated signals. Subsequently, the transit-time differences are compared against the ground-truth resulting from the reference measurement device and are represented as a relative estimation error $E(T)$ in percent.

As every signal package contains a different arrangement of the measurement signals, the estimated interfering signals also differ for each signal package which leads to the results depicted in Fig. 3. Note that the interfering signals were only estimated in the time range where the direct signals are supposed to be. The temperature-dependent relative error $E(T)$ of the estimated flow velocity is obtained from the transit-time estimation of the original signals. The relative error is influenced by the level of interfering signals and significantly reduced by our compensation, as it can be easily seen. The residual error can be explained by the model error of the non-static interfering signals as described above. An example pair of the recorded measurement signals and the different estimations of the interfering signals can be seen in Fig. 4. The fact that the estimated interfering signals have a slightly drifting time-delay also implies that only the static part can be estimated while the non-static interfering signal can not yet be separated.

\section{CONCLUSION}

A new method to separate highly-correlated interfering signals in ultrasonic flow measurements has been introduced
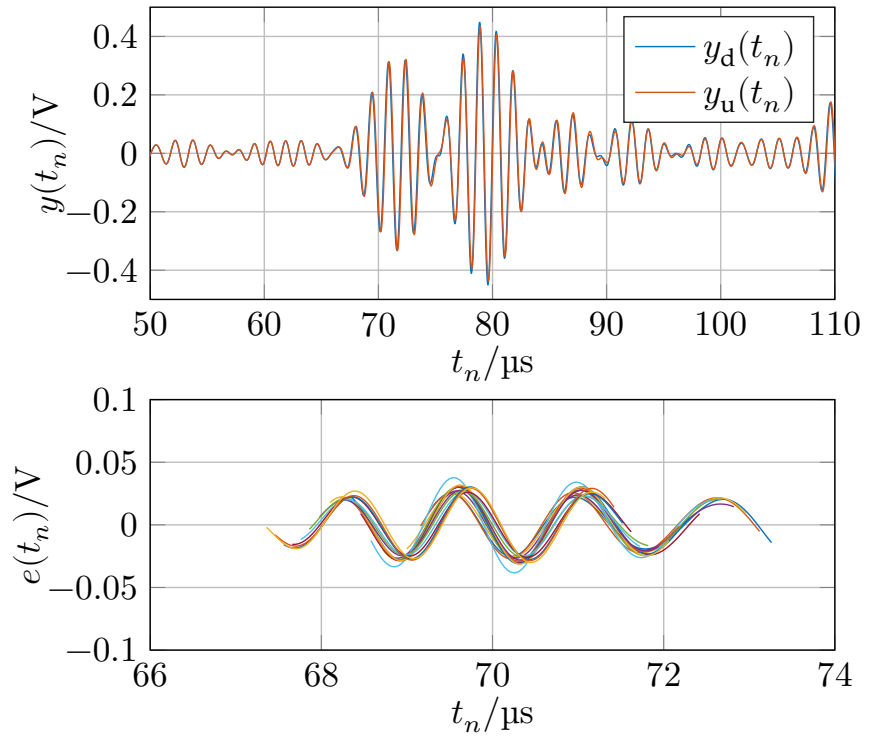

Fig. 4. Measurement signals (top) and the estimated interference signals if different temperature ranges were used as consecutive measurements (bottom).

and evaluated on simulated as well as experimental signals. The simulation results showed that the estimation quality is dependent of the noise level and the dynamics of the timedelays. If the signal model holds, the quality is sufficient to get a flow measurement accuracy better than $\pm 1 \%$. The experimental results underline these finding, even though the accuracy is limited by the non-static interfering signals.

\section{ACKNOWLEDGMENT}

We commemorate our recently deceased friend, colleague and mentor Fernando Puente León, who made this work possible with his preparatory work and his pioneering ideas.

\section{REFERENCES}

[1] L. C. Lynnworth and Y. Liu, "Ultrasonic flowmeters: Half-century progress report, 1955-2005," Ultrasonics, vol. 44, no. Suppl. 1, pp. e1371-e1378, 2006.

[2] E. Mandard, D. Kouame, R. Battault, J.-P. Remenieras, and F. Patat, "Methodology for developing a high-precision ultrasound flow meter and fluid velocity profile reconstruction," IEEE Transactions on Ultrasonics, Ferroelectrics, and Frequency Control, vol. 55, no. 1, pp. 161-172, 2008.

[3] S. J. Rupitsch, Piezoelectric Sensors and Actuators. Heidelberg: Springer-Verlag Berlin Heidelberg, 2018.

[4] N. Roosnek, "Novel digital signal processing techniques for ultrasonic gas flow measurements," Flow Measurement and Instrumentation, vol. 11, no. 2, pp. 89-99, 2000.

[5] S. A. Jacobson, P. N. Denbigh, and D. E. H. Naudé, "A new method for the demodulation of ultrasonic signals for cross-correlation flowmeters," Ultrasonics, vol. 23, no. 3, pp. 128-132, 1985.

[6] M. Bächle and F. Puente León, "Unterdrückung stationärer Interferenzsignale bei Ultraschalllaufzeitmessungen mithilfe der Hauptkomponentenanalyse/suppression of stationary interference signals in ultrasonic transit-time measurements using the principle component analysis," tmTechnisches Messen, vol. 86, no. s1, pp. 52-56, 2019.

[7] W. Wang, H. Pottmann, and Y. Liu, "Fitting b-spline curves to point clouds by curvature-based squared distance minimization," ACM Transactions on Graphics, vol. 25, no. 2, pp. 214-238, 2006.

[8] M. Kupnik, E. Krasser, and M. Gröschl, "Absolute transit-time detection for ultrasonic gas flowmeters based on time and phase domain characteristics," in 2007 IEEE Ultrasonics Symposium Proceedings, Conference Proceedings, pp. 142-145. 